\title{
Effect of Transmission Power Control on the MAC Layer of Wireless Sensor Nodes
}

\author{
J. A. Braimoh, A. M. S. Tekanyi, B. G. Bajoga \\ Department of Communications Engineering, Ahmadu Bello University, Zaria, Nigeria.
}

\begin{abstract}
The need for long term operation of large scale Wireless Sensor Networks (WSNs) has posed serious issues on energy efficiency due to the limited energy capacity of sensor nodes. Numerous schemes employed in literature have shown that the problem of effective energy management is as a result of inappropriate cluster sizes, energy consumed during data transmission by the nodes' power amplifiers, and the energy consumed at the Medium Access Control (MAC) layer of nodes for data reception. The investigation of the effect of Transmission Power Control (TPC) on the MAC layer of a self-organized cluster of wireless sensor nodes using typical CC2420 transceiver parameters, IEEE 802.15.4 standard compliant, is presented in this study. The proposed approach offers a new and more efficient method of evaluating the effect of TPC in a self-organized clustering network at the MAC layer. The network's lifetime simulation results using MATLAB R2013b, were compared when the unwanted signal received at the MAC layer of sensor nodes was considered and when not considered. The results obtained showed a $14.62 \%$ decrease in energy consumption of the network nodes, without considering the unwanted signal received at the MAC layer of the nodes.
\end{abstract}

KEYWORDS: Transmission power control, MAC layer, Wireless sensor nodes, Energy efficiency, Network lifetime.

[Received May 1, 2018; Revised August 2, 2018; Accepted September 19, 2018]

Print ISSN: 0189-9546 | Online ISSN: 2437-2110

\section{INTRODUCTION}

Recent technological advances have made it possible to deploy small, low power, low-bandwidth, and multi-functional wireless sensor nodes to monitor and report conditions and events in their local environments. A large collection of these sensor nodes can form an ad-hoc wireless sensor network, creating new types of information systems (Liu and Ning, 2007). Every sensor node in a sensor network has one or a few sensing components to sense conditions (such as temperature, humidity, pressure, etc.) from its immediate surroundings, a processing component to carry out simple computation on the raw data and a communication component to communicate with its neighbor nodes (Akyildiz et al, 2002; Oreku and Pazynyuk, 2012). WSN is used for applications such as: building automation, industrial control and monitoring, military application, medical application, traffic control, security purpose, environmental control, etc. (Recayte, 2012). However, a critical issue in the design of WSNs is how to effectively utilize its limited resources such as energy, bandwidth, storage space, processing capacity, transmission range, etc. (Zain-ul and Rauf, 2014) which would help to increase the lifetime of the network.

The lifetime of a sensor node depends basically on two factors: how much energy it consumes over time and how much energy is available for its use (Dietrich and Dressler, 2009). Network lifetime is the period until a certain number of sensor nodes are all discharged of their energy (Lee and Lee, 2013). A period is a cycle of the process of collecting data packets from the network nodes by the $\mathrm{CH}$ and transmitting the

*Corresponding author: jinadu.braimoh@gmail.com collected data to the BS. The number of repeated periods that occur during a data transmission phase before another splitting or merging cluster phase is implemented is the clustering round. The implementation of a splitting or merging cluster phase marks another clustering round.

\section{A. Review of Related Works}

A topology-controlled adaptive clustering protocol was employed in (Dahnil et al, 2012) to increase the lifetime of WSNs and maintain a required network connectivity. The proposed scheme allowed $\mathrm{CHs}$ to adjust their power level to achieve optimal degree of connectivity and maintain this value throughout the network on every periodic update. However, the energy consumed at the MAC layer of the nodes for receiving data was not considered. The impact of transmission power control in WSNs was studied in (Lina et al, 2013). In a star network, it was discovered that reducing transmission power can decrease the total energy consumption on other sensor nodes in the network. It was then concluded that TPC can save energy in a network not through reducing a transmitting sensor's cost, but through reducing other sensors' physical and MAC layer. However, the impact of MAC layer in a self-organizing network of multiple nodes deployment scenario was not considered. A Two-Tiered Topology Control (TTTC) protocol that combined clustering and power control approaches towards topology control of wireless networks was developed in (Mir et al, 2014). TTTC operation was divided into two phases. In the first phase, a clustering algorithm was executed to obtain clusters of varying sizes. In the second phase, each $\mathrm{CH}$ ran a local Minimum Spanning Tree (MST) 
based power control algorithm that helped to control the transmission power of CMs. The minimum and maximum number of CMs in a cluster was not defined which could have led to a $\mathrm{CH}$ having too many CMs. This could cause the $\mathrm{CH}$ to discharge faster. A global power management approach for energy harvesting sensor nodes that utilized a joint duty-cycle optimization and transmission power control was implemented in (Castagnetti et al, 2014). Duty-cycle management dealt with the control of task activation rate where a node followed a sleep-wake up cycle in order to balance the energy that is harvested and the energy that is consumed. TPC dealt with the RF transmission power adjustment of the node for quality packet reception at the base station. However, the energy consumed at the MAC layer of the nodes for receiving data was not considered.

Also, protocols for the MAC layer of WSNs have been studied extensively over the years. A new MAC protocol that used an improved variant of Carrier Sense Multiple Access (CSMA) and Weak Signal Detection (WSD) was implemented by (Richert et al., 2017). CSMA/WSD enabled dividing collisions from weak signals and took appropriate decisions to reduce energy consumption. CSMA/WSD as a contentionbased protocol, allowed more throughput by performing a loss diagnosis. A new QoS Medium Access Control (MAC) protocol, "PRIority in Node" (PRIN), was developed by (Subramanian \& Paramasivam, 2017) using static priority in the source and the intermediate node and among the nodes which are one hop from the sink node. By allocating different priorities to incoming packets according to the arrival priority queue of the batch, the amount of energy consumed was minimized. Throughput was increased by varying the interarrival time. An ED-Based Enhanced Energy Efficient Cross Layer Model for Mobile WSN was implemented by (Pandey \& Agrawal, 2017). Control overhead reduction during route discovery and dynamic adjustment of transmission power was used to improve network performance and reduce energy consumption. Minimization of control overhead was done by giving weights to each node. A weight was calculated by considering 'ED', associated with each node to reduce control overhead. ' $E$ ' stands for energy while ' $D$ ' stands for degree. The use of a low-power wake-up radio in WSNs was considered by (Djiroun \& Djenouri, 2017), where relevant Medium Access Control (MAC) solutions were studied. Wake-up MAC protocols were grouped into three main categories: duty cycled wake-up MAC protocols, non-cycled wake-up protocols, and path reservation wake-up protocols. These categories were discussed and analyzed. The investigation of the suitability of several WSNs MAC protocols for low data rate Wireless Multimedia Sensor Networks (WMSNs) was carried out by (AlSkaif et al., 2017). This was done by analyzing the effect of some network parameters, such as the sampling rate and the density of multimedia sensors on the energy consumption of nodes. A general multi-class traffic model that allows the integration of different types of sensors with different sampling rates was developed. Also, a mathematical modeling and a numerical evaluation of MAC protocols in WMSNs was carried out. A comparison between the energy efficiency of Video Transmission Rate Control Algorithm (VTRCA) and Transmission Power Control (TPC) algorithm by considering generic energy consumption model in Wireless Body Sensor Networks (WBSNs) was studied by (Sodhro et al., 2018). The comparison revealed how the average current $(\mathrm{mA})$ and the average data rate (bps) at the transmitter node achieved energy efficiency of VTRCA and TPC algorithms. A mathematical proof in which VTRCA outperformed TPC algorithm was presented. A Receiver-Initiated asynchronous Multichannel MAC protocol for WSN (RITMC) was proposed by (Fernandes et al., 2018). RITMC mitigated message containment and effect of Idle Listening through an initial recognition mechanism proposed by A-MAC (A versatile and efficient receiver-initiated link layer for low-power wireless) protocol.

It is evident from literature that improving the Quality of Service (QoS) of WSNs is by improving the network availability through reducing energy consumption. Many models have been proposed to reduce energy consumption in WSNs without much consideration for the unwanted signal received at the MAC layer of nodes within the transmission region. Therefore, this research is aimed at simulating the parameters of a CC2420 transceiver for the determination of the effect of TCP on the unwanted signal received at the MAC layer of a self-organized cluster of sensor nodes. This test was carried out on an existing model of Energy-Efficient SelfOrganized Clustering with Splitting and Merging (EECSM) for WSNs by Lee and Lee (2013).

\section{B. Transmission Power Control}

Transmission Power Control (TPC) is based on techniques through which a transmitter can dynamically adjust the transmission output power to the minimum or maximum level, depending on the receiver proximity (Meghji and Habibi, 2011). This allows nodes that are closer to CHs to transmit at a reduced range than the nodes farther away from the $\mathrm{CHs}$, while still preserving an acceptable received signal strength. TPC is one of the promising and effective techniques for minimizing interference and improving sensor nodes' energy consumption (Meghji and Habibi, 2011). This work is based on IEEE 802.15.4 MAC layer and implemented an adaptive TPC technique using the simulated parameters of CC2420 transceiver.

The CC2420 radio transceiver is IEEE 802.15.4 compliant and supports 8 different transmission power levels which provide a digital Received Signal Strength Indicator (RSSI) that may be read anytime. Some of the properties of the CC2420 include a data rate of $250 \mathrm{kbps}$, receiver sensitivity of $-94 \mathrm{dBm}$, frequency of $2.45 \mathrm{GHz}$, current consumption receive mode of $18.8 \mathrm{~mA}$, as well as the different power levels (Texas Instrument, 2007).

The fact that a node listens to transmitted packets on the MAC layer even when these packets are not addressed to it is called overhearing. It is energy costing since the nodes will spend energy to identify these packets to be sure that they are not addressed to it before discarding them. When a sensor's 
transmission power is reduced, the local node density is reduced by the reduction of transmission range. Some of the other sensors in the network may not hear this sensor any more. Hence overhearing is mitigated (Lina et al, 2013). By reducing a sensor's transmission power, the transmission energy consumed by a node is reduced and the number of unwanted signal received at the MAC layer of neighbor nodes which could have been processed is reduced. Thus, the total energy consumption of the network is reduced.

However, during a breakdown of a node, other nodes in the network are unable to detect its breakdown due to the lack of signal interchange between them. This affects the selforganizing ability of the network since some knowledge of the network may be unknown. The flow chart of the adaptive TPC is shown in Figure 1 which is embedded in item B of Figure 2.

Assuming a direct line of sight, the Friis free space model is used to determine distances in this work. This model is expressed mathematically as:

$$
P_{r}(d)=\frac{P_{t} G_{t} G_{r} \lambda^{2}}{(4 \pi)^{2} d^{2}}
$$

where:

$\mathrm{P}_{\mathrm{r}}(\mathrm{d})$ is the received power in $\mathrm{dBm}$ for a transmitterreceiver separation distance, $d$.

$G_{t}$ and $G_{r}$ are the transmit antenna and receive antenna gains; $G_{t}=G_{r}=1.0$

$\mathrm{d}$ is the distance between the transmitter and the receiver in meters.

$\lambda$ is the wavelength of the carrier signal in meters.

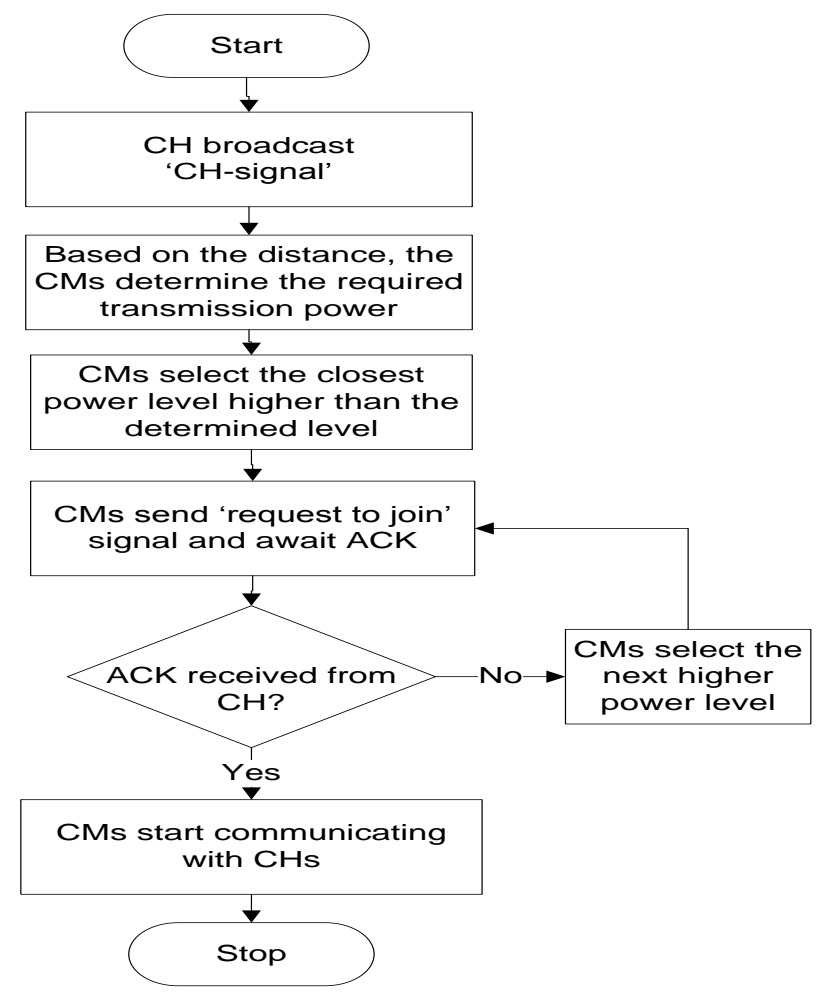

Figure 1: Flowchart for the Communication Process between the CHs and the CMs.

\section{Radio Energy Dissipation Model}

It is assumed that a constant amount of energy is consumed in the internal processing of a packet and in the transmitter amplifier. Whereas the energy consumed in amplifying the signal to achieve acceptable signal to noise ratio at a receiver is proportional to the square of the distance between transmitter and the intended receiver (Patel et al, 2004). Thus, the energy in joules required for a node to transmit a packet of length $\mathrm{k}$ bits over a distance $\mathrm{d}$ is (Liao and Zhu, 2013):

$$
\mathrm{E}_{\mathrm{Tx}}(\mathrm{k}, \mathrm{d})=\mathrm{E}_{\text {elec }} \mathrm{k}+\varepsilon_{\mathrm{amp}} \mathrm{kd}^{2}
$$

The energy in joules consumed at the receiving node is (Liao and Zhu, 2013):

$\mathrm{E}_{\mathrm{Rx}}(\mathrm{k})=\mathrm{E}_{\text {elec }} \times \mathrm{k}$

where:

$\mathrm{E}_{\mathrm{Tx}}(\mathrm{k}, \mathrm{d})$ is the energy consumption in transmitting $\mathrm{k}$ bits data to a node with a distance of $d$. data.

$\mathrm{E}_{\mathrm{Rx}}(\mathrm{k})$ is the energy consumption in receiving $\mathrm{k}$ bits

$\mathrm{E}_{\text {elec }}$ is the per bit energy consumption for transmitter and receiver circuitry

$\varepsilon_{\text {amp }}$ is the per bit energy consumption by the transmitter amplifier

$k$ is the packet data size (1,000 bits)

\section{Parameters used in the Simulation}

The parameters in Table 1 were considered in this work:

i. The locations of all sensor nodes and the BS are fixed.

ii. The deployment of sensor nodes uses random distribution.

iii. It is assumed that the WSN cannot operate when $30 \%$ of the sensor nodes are discharged of energy.

iv. Cluster heads directly transmit the data packets received from their $\mathrm{CM}$ s to the $\mathrm{BS}$.

Table 1: Parameters used in the Simulation.

\begin{tabular}{ll}
\hline Parameter & Value \\
\hline Sensor field & $250 \mathrm{~m}$ by $250 \mathrm{~m}$ \\
Location of Base Station & $x-$ axis: $300 \mathrm{~m}$, \\
& $y-$ axis: $300 \mathrm{~m}$ \\
Broadcasting range & $50 \mathrm{~m}$ \\
Packet data size $(\boldsymbol{k})$ & $1,000 \mathrm{bits}$ \\
Initial energy of sensor nodes & $0.5 \mathrm{~J}$ \\
Number of deployments & 10 \\
Number of sensor nodes & 100 \\
The per bit energy consumption for transmitter & $50 \mathrm{~nJ} / \mathrm{bit}$ \\
and receiver circuitry $\left(\mathbf{E}_{\text {elec }}\right)$ & \\
The per bit energy consumption by the node's & $100 \mathrm{pJ} / \mathrm{bit} / \mathrm{m}^{2}$ \\
amplifier $\left(\boldsymbol{\varepsilon}_{\text {amp }}\right)$ & \\
Splitting and merging threshold & 46 and 20 nodes \\
& respectively \\
\hline
\end{tabular}

\section{EXPERIMENTAL PROCEDURE}

The steps of the methodology adopted for this research towards simulating the effect of TPC on the signal received at the MAC layer in a self-organized clustering of sensor nodes are explained in Figure 2. 


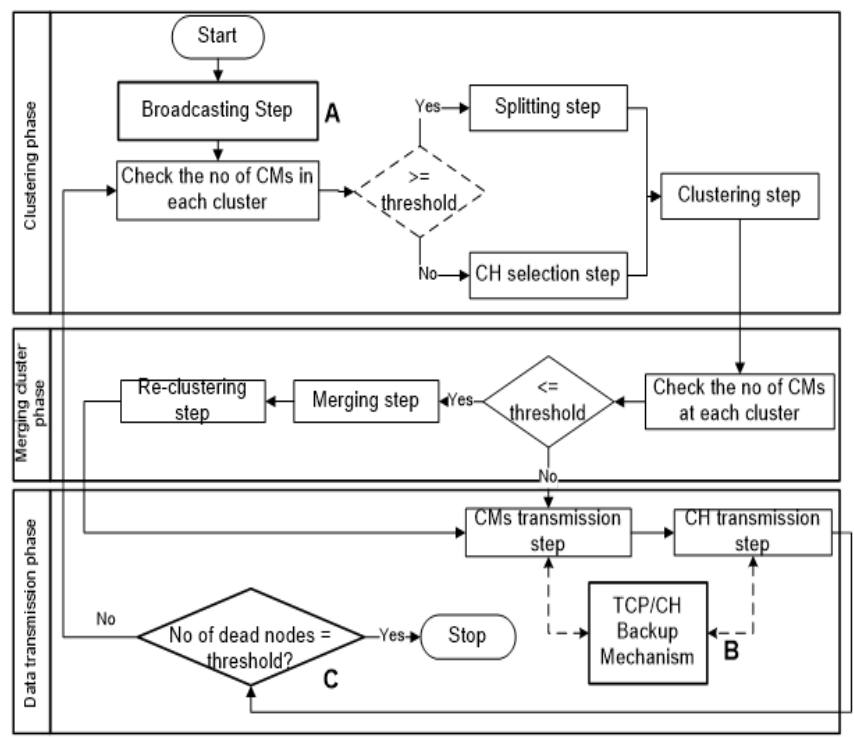

Figure 2: Flowchart for the Modified EECSM with TPC.

This flowchart was modified from the work of Lee and Lee (2013). The dotted bidirectional arrows in Figure 2 indicates the flow of control messages between the CMs and $\mathrm{CHs}$ while the solid arrow between the $\mathrm{CMs}$ and the $\mathrm{CH}$ indicates a direct communication link through which the sensed information is transmitted from the $\mathrm{CMs}$ to the $\mathrm{CH}$.

\section{A. Clustering/Self-Organizing Phase}

Sensor nodes are deployed in a geographical area where they organize themselves to form a network of their own. This typically involves the decomposition of the network into clusters of bounded sizes with each cluster having a $\mathrm{CH}$ and many CMs. The clustering phase commences when the sensor nodes are first scattered in the sensor field or when the next $\mathrm{CH}$ is activated. The clustering phase is comprised of three steps: broadcasting step, splitting/CH selection step, and clustering step.

1) Broadcasting Step: At initial deployment, there are no CHs. Every node in the network competes for the position of a $\mathrm{CH}$ by broadcasting information about its "sensor ID" and "energy level" to nodes within its broadcasting range of 10 meters. Any node that receives broadcasted energy level higher that its own automatically stops competing for the position of a $\mathrm{CH}$ and tries to connect with the node that reported higher energy level which then becomes the First $\mathrm{CH}$. In case of a tie in energy level of nodes, the number of neighbors within the broadcasting range of a node is considered. That is, the node having the most neighbors is selected as the First $\mathrm{CH}$. The First $\mathrm{CH}$ broadcast "CH-signal" packets and all the nodes in the network get connected to it.

2) Splitting Cluster/CH Selection Step: If the number of nodes connected to the First $\mathrm{CH}$ is more than the splitting threshold, it partitions the network into smaller clusters by selecting the Second $\mathrm{CH}$ based on CMs' residual energy level. Should a cluster exceed the splitting threshold, the $\mathrm{CH}$ of that particular cluster further breaks the network into smaller clusters by selecting the Third $\mathrm{CH}$.

For every clustering round, a $\mathrm{Next} \mathrm{CH}$ is selected by the $\mathrm{CHs}$ for their respective clusters. The $\mathrm{Next} \mathrm{CH}$, that is the $\mathrm{CM}$ having maximum residual energy of the cluster, is activated for the next clustering round.

3. Clustering Step: The selected $\mathrm{CHs}$ then broadcast " $\mathrm{CH}$ signal" packets and all the nodes in the network get connected to the $\mathrm{CH}$ closer to them by comparing their distances from both $\mathrm{CHs}$ using the RSSI of the received signal. The $\mathrm{CHs}$ receive acknowledgements from the $\mathrm{CMs}$ and add them to its cluster.

\section{B. Merging Cluster Phase}

A CH broadcasts a 'merging-cluster signal' to the nodes when the number of nodes it has is less than or equal to the merging threshold which may be due to breakdown of nodes after a long operational period of the network. All the nodes initially connected to that $\mathrm{CH}$, realizing they no longer have a $\mathrm{CH}$, connect to a $\mathrm{CH}$ closer to them by sending 'request to join' signal packet. The $\mathrm{CH}$ that receives this message sends ACK and adds them to its cluster. The $\mathrm{CH}$ that broadcasted the 'merging-cluster signal' also gets connected to a closer $\mathrm{CH}$ by sending 'request to join' signal packet.

\section{Data Transmission Phase and CH Backup Mechanism}

Once the merging cluster phase is completed, clustering is accomplished. The EECSM enters into the data transmission phase. In the data transmission phase, information gathered from the sensor field is sent to the $\mathrm{CHs}$ which in turn aggregates the information and relay it to the BS.

When the CMs recognize a breakdown of their $\mathrm{CHs}$ during the data transmission phase, $\mathrm{CH}$ reelection step is carried out immediately. All the CMs broadcast their energy state signal within the broadcasting range twice to elect a new $\mathrm{CH}$. The $\mathrm{CM}$ having maximum residual energy becomes the new $\mathrm{CH}$, which then broadcasts the "CH-signal" to the entire area of the sensor field enabling the sensor nodes to decide their $\mathrm{CH}$ according to distance.

\section{CC2420 Transmission Power Levels and Average Distance using Friis Equation}

Table 2 shows the average transmission distances that can be covered for the different transmission power levels of the CC2420 transceiver using the Friis equation. Where the received power is taken as the receiver sensitivity with a transmit power of $0 \mathrm{dBm}$ (Meghji and Habibi, 2011) and a frequency of $2.45 \mathrm{GHz}$, assuming no transmission losses. The CMs are designed to select the power level higher than the determined power level. This helps to give an acceptable signal reception at the receiver. 
Table 2: CC2420 Transmission Power Levels and

Average Distance.
\begin{tabular}{|c|c|c|c|}
\hline $\begin{array}{c}\text { Power } \\
\text { Levels }\end{array}$ & $\begin{array}{c}\text { Transmission } \\
\text { Power (dBm) }\end{array}$ & $\begin{array}{c}\text { Current } \\
\text { Drained } \\
(\mathbf{m A})\end{array}$ & $\begin{array}{c}\text { Distance }(\mathbf{m}) \\
\left(\boldsymbol{R}_{\boldsymbol{x}}=-\right. \\
\mathbf{9 4 d B m})\end{array}$ \\
\hline 31 & 0 & 17.4 & 488.4 \\
\hline 27 & -1 & 16.5 & 435.3 \\
\hline 23 & -3 & 15.2 & 345.7 \\
\hline 19 & -5 & 13.9 & 274.6 \\
\hline 15 & -7 & 12.5 & 218.2 \\
\hline 11 & -10 & 11.2 & 154.4 \\
\hline 7 & -15 & 9.4 & 86.9 \\
\hline 3 & -25 & 8.5 & 27.5 \\
\hline
\end{tabular}

The transmission power levels shown in Table 2 is converted into energy (Meghji and Habibi, 2011). The amplifier energy consumption $\left(E_{a m p}\right)$ for a selected power level $(\mathrm{x})$ is represented as:

$E_{a m p}(x)=[P(x) \times t]$

where:

$E_{a m p}$ is the amplifier energy consumption for a selected power level $(x)$ in joules.

$P(x)$ is the power consumed at a power level $(x)$ with a voltage of $1.8 \mathrm{~V}$ (Texas Instrument, 2007).

Time $(\mathrm{t})=\frac{1}{R_{b}}$ seconds $\left(4 \times 10^{-6}\right.$ seconds $)$ is the time it takes to transmit 1 bit of data

$R_{b}$ is the data rate of $250 \mathrm{kbps}$ (Texas Instrument, 2007).

Since the energy consumed by the node is made up of both the constant electronic energy consumption and the energy consumed by the radiating power amplifier, from equation (2), the total node energy consumption becomes:

$$
\mathrm{E}_{\mathrm{Tx}}(\mathrm{k}, \mathrm{d})=\left[\mathrm{E}_{\mathrm{elec}}+E_{a m p}(x)\right] k
$$

Also, the energy consumed for data reception at the MAC layer of the radio transceiver is determined by taking into consideration the current it consumes for signal reception. The value of this Current $(I)$ is given as $18.8 \mathrm{~mA}$ (Texas Instrument, 2007). Thus, the energy consumed at the receiving node can be modeled from equation (3) as the sum of energy consumption for the receiver circuitry and the energy consumed for signal received at the MAC layer $\left(\mathrm{E}_{\mathrm{mac}}\right)$ as:

$$
\mathrm{E}_{\mathrm{Rx}}(\mathrm{k})=\left[\mathrm{E}_{\mathrm{elec}}+\mathrm{E}_{\mathrm{mac}}\right] \mathrm{k}
$$

\section{RESULTS AND DISCUSSION}

Simulations were carried out according to the flowchart of Figure 2. Results for network lifetime of WSNs using the parameters of CC2420 radio transceiver were obtained and used to validate the effect of TPC on the signal received at the MAC layer of sensor nodes in the network. The network lifetime against number of dead sensor nodes was plotted for 100 nodes in a field of $250 \mathrm{~m}^{2}$.

\section{A. Comparison of Network Lifetime with and Without Consideration for the Unwanted Signal at the MAC Layer of Nodes with TPC}

From Figure 3, when the unwanted signal received at the MAC layer of nodes was considered in a self-organizing network that utilized TPC (that is, when the energy consumed at the MAC layer to process the unwanted received signal was added in the simulation), it took an average network lifetime of 1481 periods for the 30th node to be discharged of its energy. Also, when the unwanted signal received at the MAC layer of the nodes was not considered in the same network, an average network lifetime of 2599 periods for the 30th node to be discharged of its energy was recorded. From the result, it was found that a high amount of energy was consumed by the node in processing the unwanted signal received at its MAC layer during data transmission.

When the network was operated using TPC technique, there was $43.0166 \%$ improvement of network lifetime without considering the unwanted signal received at the MAC layer. Meaning that $56.9835 \%$ of the energy consumed in the network was by sensor nodes receiving data signal that is not meant for them at the MAC layer and this reduced the network lifetime.

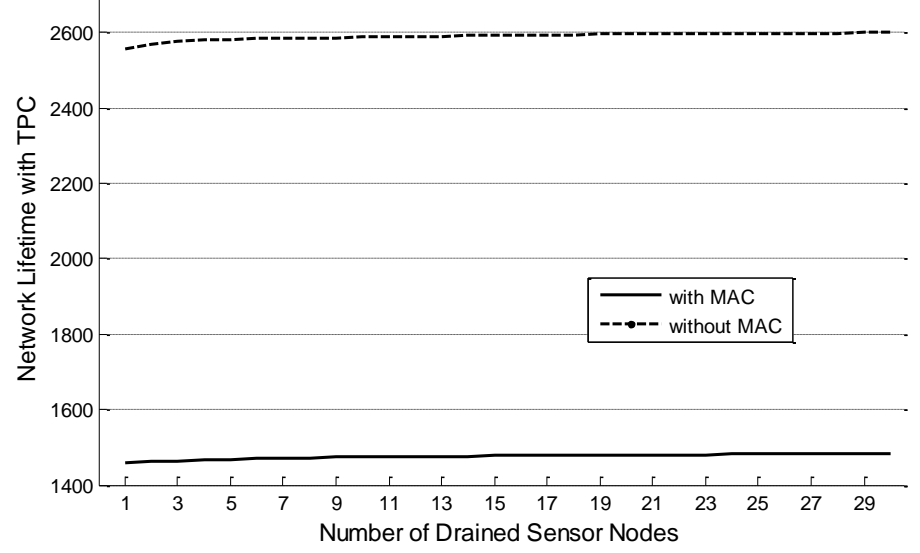

Figure 3: Network Lifetime with and without Consideration for the Unwanted Signal received at the MAC Layer using TPC.

Also, it is seen from the graph that the network lifetime increased faster at the beginning of the plot than towards the end of the plot. This is because it gets to a point where the Residual Energy (RE) of some nodes in the network become so low that they are unable to complete their tasks before their energy level become zero due to the balanced nature of the network load. At this point (after the discharge of 15 nodes approximately), the nodes die faster and almost simultaneously. Hence, there is no more increase in the network lifetime.

Note: The network is assumed to fail when 30 nodes are discharged of energy as stated in section 1.4.

\section{B. Comparison of Network Lifetime With and Without Consideration for the Unwanted Signal at the MAC layer of Nodes without TPC}

From Figure 4, when the signal received at the MAC layer of nodes was considered in a self-organizing network without the use of TPC (that is, when the energy consumed by the node for receiving an unwanted signal at its MAC layer was added in the simulation without utilizing TPC), it took an average network lifetime of 1287 periods for the 30th node to be discharged of its energy. Also, when the unwanted signal received at the MAC layer was not considered in the same selforganizing network without TPC, an average network lifetime of 2034 periods for the 30th node to be discharged of its energy 
was recorded. From the result, it is found that a higher amount of energy is consumed by the nodes in processing the unwanted signal received at the MAC layer during data transmission when TPC was excluded from the network.

When the network was operated without TPC technique, there was $36.7257 \%$ improvement of network lifetime without considering the unwanted signal received at the MAC layer. Meaning that $63.2744 \%$ of energy was consumed by nodes receiving data that is not meant for them in their MAC layer which reduces the network lifetime.

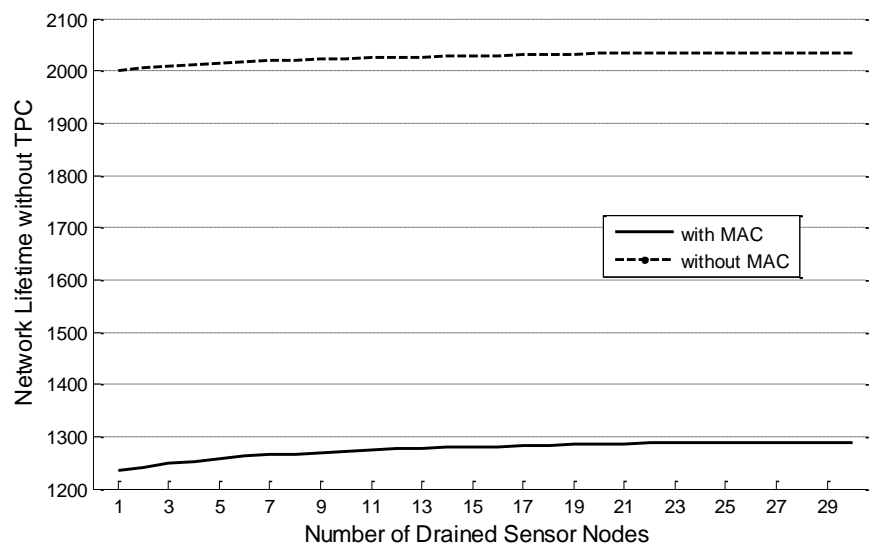

Figure 4: Network Lifetime with and without Consideration for the Unwanted Signal received at the MAC layer.

\section{Summary}

When the network was operated without considering the unwanted signal received at the MAC layer, there was $43.0166 \%$ and $36.7257 \%$ improvement of network lifetime with and without the use of TPC technique respectively. This indicates that TPC saved up to $14.6244 \%$ of energy that could have been wasted on transmissions over unnecessary distances. Hence, more nodes received data at their MAC layer when TPC was not considered in the network.

By considering the energy expelled for processing the unwanted signals received at the MAC layer, a node having more neighbors within its $50 \mathrm{~m}$ radius received and decoded more signals which consumed most of its energy. With TPC, each node had to deal with a radius of less than $50 \mathrm{~m}$. This made the nodes to consume less energy at their MAC layers for processing the unwanted packets/signals.

However, with TPC, a substantial amount of energy is still used up in the network when the consumed energies at the MAC layers are summed up. Therefore, by disregarding the energy consumed at the MAC layer of nodes for receiving unwanted signal, only the energy expelled for decoding the wanted packets/signals is considered. Hence, the marginal difference between with and without consideration for the unwanted signals received at the MAC layers after a number of periods.

\section{CONCLUSION}

A study on the effect of Transmission Power Control (TPC) on the signal received at the MAC layer of a self- organized clustering of wireless sensor nodes using typical CC2420 transceiver parameters, which is IEEE 802.15.4 standard compliant, was carried out. This research offer a new approach to test TPC in a self-organized clustering network at the MAC layer and provide energy consumption performance results via simulations. It was established through simulation results that TPC can save energy in a network not only by reducing a node's transmission energy consumption, but also, by reducing the energy consumed for processing the unwanted signals received at the MAC layer of neighbor nodes. Results obtained from simulations using MATLAB R2013a showed that Network Lifetime was greatly improved by disregarding the unwanted signals received at the MAC layer of nodes in the network.

This work can be extended to consider mitigating interference among neighboring nodes by using modulation techniques such as the Time Division Multiple Access (TDMA) technique on the Cluster Member (CM) nodes to increase Network Lifetime.

\section{REFERENCES}

Akyildiz, I. F.; W.Su; Y. Sankarasubramaniam and E. Cayirci. (2002). Wireless Sensor Networks: A Survey. Journal of Computer Networks, 38(4): 393-422.

AlSkaif, T.; B. Bellalta; M. G. Zapata and J. M. B. Ordinas. (2017). Energy Efficiency of MAC Protocols in Low Data Rate Wireless Multimedia Sensor Networks: A comparative study. Ad Hoc Networks, 56:141-157.

Castagnetti, A.; A. Pegatoquet; T. N. Le and M. Auguin. (2014). A Joint Duty-Cycle and Transmission Power Management for Energy Harvesting WSNs. IEEE Transactions on Industrial Informatics, 10(2): 928-936.

Dahnil, D. P.; Y. P. Singh and C. K. Ho. (2012). Topology-Controlled Adaptive Clustering for Uniformity and Increased Lifetime in Wireless Sensor Networks. International Journal of Wireless Sensor Systems, 2(4): 318-327.

Dietrich, I. and Dressler, F. (2009). On the Lifetime of Wireless Sensor Networks. ACM Transactions on Sensor Networks, (TOSN), 5(1): 1-38

Djiroun, F. Z. and Djenouri, D. (2017). MAC Protocols with Wake-up Radio for Wireless Sensor Networks: A review. IEEE communications surveys and tutorials, 19(1): 587-618.

Fernandes, R. F.; M. B. de Almeida and D. Brandão. (2018). An Energy Efficient Receiver-Initiated MAC Protocol for Low-Power WSN. Wireless Personal Communications, 100(4):1517-1536.

Lee, K. and Lee, H. (2013). Energy-Efficient SelfOrganized Clustering with Splitting and Merging for Wireless Sensor Networks. International Journal of Distributed Sensor Networks, 9(3): 1-12.

Liao, Q. and Zhu, H. (2013). An Energy Balanced Clustering Algorithm Based on LEACH Protocol. Journal of Applied Mechanics and Materials, 341(2): 1138-1143.

Lina, X.; D. T. Declan; O. M. P. Gregory and C. Rem. (2013). The impact of transmission power control in wireless sensor networks. 12th IEEE International Symposium on 
Network Computing and Applications, Cambridge, MA, USA, 255-258.

Liu, D. and Ning, P. (2007). Security for wireless sensor networks, Springer Science and Business Media Publishing, New York, USA, 1 - 2.

Meghji, M. and Habibi, D. (2011). Transmission power control in multi-hop wireless sensor networks 3rd International Conference on Ubiquitous and Future Networks (ICUFN), Dalian, China, 25-30.

Mir, Z. H.; K. W. Lim and Y. B. Ko. (2014). On the interplay between clustering and power control in multihop wireless networks. 11th IEEE International Conference on Computer Systems and Applications (AICCSA), Doha, Qatar, 310-317.

Oreku, G. S. and Pazynyuk, T. (2016). Security in Wireless Sensor Networks, Springer International Publishing, Switzerland.

Pandey, S., and Agrawal, D. (2017). An ED-Based Enhanced Energy Efficient Cross Layer Model for Mobile Wireless Sensor Network. National Academy Science Letters, 40(6), 421-427.

Patel, M.; R. Chandrasekaran and S. Venkatesan. (2004). Efficient Minimum-Cost Bandwidth-Constrained Routing in Wireless Sensor Networks, International Conference on Wireless Networks, 447-453.

Razaque, A.; S. Mudigulam; K. Gavini; F. Amsaad; M. Abdulgader and G. S. Krishna. (2016). H-LEACH: Hybrid-low energy adaptive clustering hierarchy for wireless sensor networks. IEEE Conference on Long Island Systems, Applications and Technology (LISAT), Farmingdale, NY, USA, 1-4.

Recayte, E. (2012). A Java application to display temperature, humidity and luminosity in a Wireless Sensor Network". Corso di Laurea in Ingegneria dell'Informazione, Universita degli Studi di, Padova, 9-20.

Richert, V.; B. Issac and N. Israr. (2017). Implementation of a Modified Wireless Sensor Network MAC Protocol for Critical Environments. Wireless Communications and Mobile Computing, 1-23.

Sodhro, A. H.; L. Chen; A. Sekhari; Y. Ouzrout and W. Wu. (2018). Energy Efficiency Comparison between Data Rate Control and Transmission Power Control Algorithms for Wireless Body Sensor Networks. International Journal of Distributed Sensor Networks, 14(1): 1-18.

Subramanian, A. K. and Paramasivam, I. (2017). PRIN: a Priority-based Energy Efficient MAC Protocol for Wireless Sensor Networks varying the Sample Inter-arrival Time. Wireless Personal Communications, 92(3):863-881.

Texas instrument: Cc2420, (2007). [Online]. Available at: http://www.ti.com/product/cc2420/. Accessed on November 7, 2017.

Zain-ul, A. J. and Rauf, S. (2014). A Survey on Energy Efficient Routing Techniques in Wireless Sensor Networks Focusing on Hierarchical Network Routing Protocols. International Journal of Scientific and Research Publications, 4: 1-7. 